\title{
Brechas de género en estudiantes de la Universidad Estatal a Distancia de Costa Rica y recomendaciones para su disminución
}

\author{
Rosberly Rojas Campos, Rocío Chaves Jiménez y Ana Lucía Fernández Fernández \\ Universidad Estatal a Distancia, 474-2050 San Pedro, Montes de Oca, San José, Costa Rica; rrojas@uned.ac.cr; rchaves@uned.ac.cr; \\ afernandezf@uned.ac.cr
}

Recibido 24-I-2012 Corregido 31-V-2012 Aceptado 21-VI-2012

\begin{abstract}
Student gender gaps in the Costa Rican Distance Education University and recommendations to reduce them. Women access Higher Education, every day in greater numbers. In the Universidad Estatal a Distancia de Costa Rica (UNED), women are $60 \%$ of the student population, but enrollment does not necessarily mean more and better opportunities. Because of that, a study was done between July and September 2010 with a questionnaire applied to a random sample of 167 UNED students. We analyzed differences in studying opportunities and access to institutional services between male and female students, in order to recognize some of the students' conditions on areas like study, knowledge and gender stereotypes. There are gaps that block equitable and equal access to education opportunities on the student population, including career choice, tutoring assistance, study time, working time (paid and unpaid) and the existence of emotionally and financially dependent people. There is a clear need for an institutional policy for gender equity and equality, and a subsequent plan of action leading towards the elimination of the identified gaps through gender mainstreaming on fundamental activities like teaching, research, extension and university management.
\end{abstract}

\section{KEY WORDS}

Gender, gender gap, gender equality, gender equity, university, distance education, students.

\section{RESUMEN}

Las mujeres acceden, cada vez en mayor número, a la Educación Superior; en la Universidad Estatal a Distancia de Costa Rica (UNED) alcanzan el $60 \%$ de la población estudiantil. Sin embargo, mayor matrícula no necesariamente equivale a mayores y mejores oportunidades. Es por ello, que se analizan las diferencias existentes entre mujeres y hombres estudiantes de la UNED en las oportunidades de estudio y el acceso a los servicios institucionales. Para ello se aplicó un cuestionario al estudiantado en los meses de julio a setiembre del 2010, el cual permitió reconocer algunas de sus condiciones de estudio, conocimientos y estereotipos de género. Se presentan evidencias sobre las brechas, convertidas en acciones discriminatorias, que impiden el acceso igualitario y equitativo a las oportunidades de educación en la población estudiantil; tales como, la elección de carrera, la asistencia a las tutorías, la dedicación horaria al estudio, las jornadas de trabajo remunerado y no remunerado y la existencia de personas que dependen emocional y económicamente del estudiantado. También se muestran resultados sobre las percepciones acerca de los roles tradicionales de género en la sociedad. Se discute sobre la necesidad de formular una política institucional de igualdad y equidad de género y su respectivo plan de acción dirigido hacia la eliminación de las brechas identificadas, por medio de una estrategia de transversalización de la perspectiva de género en las actividades fundamentales de docencia, investigación, extensión y gestión universitaria.

PALABRAS CLAVE

Género, brechas de género, equidad de género, igualdad de género, universidad, educación a distancia, estudiantes.
Los sistemas discriminatorios y las condiciones de desigualdad entre mujeres y hombres se encuentran presentes en todos los ámbitos de la sociedad, la mayor parte de las veces pasan inadvertidos y se asumen como normales y naturales sin posibilidad de modificación (Calvo, 1990). Las diferencias sexuales por sí mismas no provocan desigualdad, pero cuando la sociedad le asigna un mayor valor a alguna de esas diferencias, la desigualdad resultante impide que ambos sexos tengan el mismo acceso a oportunidades para su desarrollo personal y colectivo (Lagarde, 1996). Esto produce brechas de género, entendidas como "las diferencias existentes entre mujeres y hombres 
en relación con las oportunidades, el uso, acceso y control de los recursos y los servicios que limitan el ejercicio de sus derechos humanos, su bienestar y desarrollo integral" (Instituto Nacional de las Mujeres, 2002, p.7).

Género es una construcción social que hace referencia a los aspectos no biológicos del sexo y, por ende, puede ser modificada (Stoller, 1968; Oakley, 1972; Rubin, 1975; Money \& Tucker, 1978). Una manera de promover estos cambios en la cultura es por medio de la promoción de políticas públicas que pongan en marcha acciones y estrategias específicas para la eliminación de las brechas de género identificadas y para la construcción de una sociedad más igualitaria e inclusiva.

Dado lo anterior, Costa Rica ha suscrito y ratificado instrumentos internacionales de gran relevancia como la Convención sobre la Eliminación de todas las formas de Discriminación contra la Mujer, conocida como CEDAW por sus siglas en inglés, y la Plataforma de Acción de Beijing, emanada de la IV Conferencia Mundial de la Mujer celebrada en China en el año de 1995. En ambos instrumentos se reconoce el compromiso que los Estados deben asumir en la promoción de una educación no discriminatoria y en garantizar las mismas oportunidades de desarrollo tanto para mujeres como para hombres.

Los avances en la aplicación de políticas de género en Costa Rica han llevado a situar al país en el puesto número 28 del Índice Global de Brecha de Género (Hausmann et al., 2010). En cuanto a los componentes del índice, se tiene una posición ventajosa respecto del Empoderamiento Político al conseguir el puesto 14, pero se desmejora en relación con Participación y Oportunidades Económicas (puesto 98), Salud y Sobrevivencia (puesto 66) y Logro Educativo (puesto 46) (Hausmann et al., 2010, p.10). La matrícula en Educación Terciaria, que está contemplada como uno de los componentes del subíndice Logro Educativo, presenta una razón entre sexos (mujeres/hombres) de 1.26 (Hausmann et al., 2010, p.108) con lo cual se evidencia un mayor acceso de parte de las mujeres.

En general, en el mundo se han reducido las brechas de acceso a la Educación Superior entre mujeres y hombres (The World Bank, 2011, p.107); sin embargo, otras brechas aún persisten, tal es el caso del ingreso a las diferentes disciplinas, donde mayormente los hombres continúan estudiando ciencias naturales, matemáticas e ingeniería, mientras las mujeres continúan estudiando educación, artes, humanidades, ciencias sociales y leyes (Bradley, 2000; The World Bank, 2011). En este sentido, sigue siendo válido profundizar en las condiciones de estudio y los servicios obtenidos por la población estudiantil en la Educación Superior, que le permitan obtener su máximo potencial en la sociedad (Bonder, 1994). Es por ello que, este artículo tiene como objetivo analizar las brechas existentes entre mujeres y hombres estudiantes de la Universidad Estatal a Distancia (UNED) en las oportunidades de estudio y el acceso a los servicios institucionales y los posibles contextos generadores de discriminación.

\section{METODOLOGÍA}

Se elaboró y aplicó un cuestionario al estudiantado de la UNED, en los meses de julio, agosto y setiembre del 2010, el cual pretendió identificar características poblacionales generales, reconocer algunos estereotipos sexistas y conocimientos sobre género en la UNED.

De las 21355 personas que realizaron matrícula ordinaria en las carreras de grado y postgrado de la UNED en el I cuatrimestre del 2010, de acuerdo al Sistema de Administración de Estudiantes de la Oficina de Registro, se incluyeron en el marco muestral 15889 estudiantes regulares. Las restantes 5466 personas, que correspondían a estudiantes de primer ingreso (matriculan por primera vez en la UNED) no fueron incluidas, dado que apenas se inician en la modalidad a distancia.

A partir del marco muestral, se realizó una selección aleatoria utilizando la fórmula de Mendenhall (1987), con un error de estimación del $5 \%$ y un valor de $\mathrm{P}=0,88$ como porcentaje de la población que cumple con la característica de interés. El tamaño de la muestra fue de 167 estudiantes.

El cuestionario se aplicó por teléfono, con una respuesta del 82,64\% (138 en total). La información obtenida fue procesada con Statistical Package for the Social Science SPSS para extraer las frecuencias relativas, las cuales son necesarias para realizar las mediciones de brecha entre los géneros, según la metodología del Índice Global de Brecha de Género (Hausmann et al., 2010).

La metodología citada mide las brechas en el acceso a recursos y oportunidades más que los niveles en la disponibilidad de éstos. Así, a modo de ejemplificar el concepto, el índice penaliza o retribuye a la Universidad con base en el tamaño de la brecha entre hombres y mujeres matriculados en la universidad, pero no por los niveles totales de matrícula.

La medida busca mostrar la igualdad o desigualdad entre los géneros, de acuerdo con la proximidad a 1,0 de la razón entre el porcentaje de mujeres (M) que cumplen con una determinada característica y el porcentaje de hombres $(H)$ que también la cumplen (razón $M / H$ ).

Para la estimación se utilizó una escala negativa-positiva (Hausmann et al., 2010, p. 4) tomando en cuenta el tamaño y dirección de la brecha de género. Con este tipo de escala se identifica la existencia de brecha ya sea cuando 
hay ventajas para los hombres respecto a las mujeres (razón $\mathrm{M} / \mathrm{H}<1,0$ ) ó cuando las hay para las mujeres respecto a los hombres (razón $\mathrm{M} / \mathrm{H}>1,0$ ), la no existencia de brechas se obtiene cuando la razón $\mathrm{M} / \mathrm{H}$ es igual a 1,0.

\section{RESULTADOS}

\section{Características generales de la población estudiantil}

La mayoría de la población (56,9\%) está en el rango de los 20 a 29 años, tanto para las mujeres (60,5\%), como para los hombres $(51,0 \%)$. El estado civil más común fue la soltería $(58,0 \%)$ pero si se diferencia por sexo, este es mucho más frecuente en el caso de las mujeres $(65,1 \%)$ que en el de los hombres (46,2\%). La mayoría no tienen hijos/as, tanto mujeres $(59,3 \%)$ como hombres $(53,8 \%)$ (Cuadro 1).

\section{Escogencia de carrera y áreas de estudio}

La mayoría de la población estudiantil ingresó a la UNED en los últimos cinco años, que corresponde al $83,5 \%$ de las mujeres y el $82,7 \%$ de los hombres; $14,1 \%$ de ellas y $15,4 \%$ de ellos ingresaron hace 6 a 10 años atrás y un mínimo porcentaje ( $2,4 \%$ de mujeres y $1,9 \%$ de hombres) lo hicieron hace más de 10 años.

Como se observa en el cuadro 2, las carreras más escogidas por las mujeres son las de Educación $(31,7 \%)$ y Administración de Empresas (28,0\%). Los hombres tienen preferencias de estudios en Administración de Empresas $(47,1 \%)$.

Los datos de la población entrevistada revelan que las mujeres principalmente eligen carreras en el campo de la educación, la salud, los servicios en general (turismo por ejemplo) mientras que más hombres eligen carreras como ingeniería.

Al comparar los datos de la población entrevistada con los totales de la Universidad, por Escuela, se evidencia la brecha desfavorable para las mujeres en tres de ellas, especialmente en la de Ciencias Exactas y Naturales donde la razón M/H apenas llega a 0,5 es decir, por cada 2 hombres hay una mujer. En la escuela de Educación, la brecha desfavorable es para los hombres, al matricularse más de 3 mujeres por cada hombre (Cuadro 3).

CUADRO 1

Sexo, edad, estado civil y número de hijos/as de las personas entrevistadas, por sexo, 2010.

\begin{tabular}{|c|c|c|c|c|}
\hline \multicolumn{2}{|c|}{ Características } & \multirow{2}{*}{$\begin{array}{c}\text { Total (\%) } \\
100,0\end{array}$} & \multirow{2}{*}{$\frac{\text { Mujeres (\%) }}{62,3}$} & \multirow{2}{*}{$\frac{\text { Hombres (\%) }}{37,7}$} \\
\hline Sexo & & & & \\
\hline Edad & & 100,0 & 100,0 & 100,0 \\
\hline & Menos de 20 años & 9,5 & 11,6 & 5,9 \\
\hline & 20 a 29 años & 56,9 & 60,5 & 51,0 \\
\hline & 30 a 39 años & 22,6 & 18,6 & 29,4 \\
\hline & 40 a 49 años & 9,5 & 9,3 & 9,8 \\
\hline & 50 a 59 años & 1,5 & 0,0 & 3,9 \\
\hline \multirow[t]{6}{*}{ Estado civil } & & 100,0 & 100,0 & 100,0 \\
\hline & Casada/o & 26,8 & 22,1 & 34,6 \\
\hline & Soltera/o & 58,0 & 65,1 & 46,2 \\
\hline & Unión Libre & 12,3 & 10,4 & 15,4 \\
\hline & Divorciada/o & 2,2 & 1,2 & 3,8 \\
\hline & Separada/o & 0,7 & 1,2 & 0,0 \\
\hline \multirow[t]{5}{*}{ Hijos } & & 100,0 & 100,0 & 100,0 \\
\hline & No tiene & 57,2 & 59,3 & 53,8 \\
\hline & Uno & 26,1 & 30,2 & 19,2 \\
\hline & Dos & 8,7 & 7,0 & 11,5 \\
\hline & Más de dos & 8,0 & 3,5 & 15,5 \\
\hline
\end{tabular}


CUADRO 2

Área de estudio de la población estudiantil entrevistada, por sexo, 2010

\begin{tabular}{lccc}
\hline Área de estudio & Mujeres (\%) & Hombres (\%) & Razón M/H \\
\hline Educación & 31,7 & 15,7 & 2,0 \\
Administración de Empresas & 28,0 & 47,1 & 0,6 \\
Estudios Generales & 11,0 & 7,8 & 1,4 \\
Salud & 7,3 & 5,9 & 1,2 \\
Turismo & 6,1 & 5,9 & 1,0 \\
Recursos naturales & 4,9 & 2,0 & 2,5 \\
Programas de posgrado & 3,7 & 2,0 & 1,9 \\
Ingeniería Informática & 2,4 & 5,9 & 0,4 \\
Otras' & 4,9 & 7,7 & 0,6 \\
Total & 100,0 & 100,0 & - \\
'En la categoría Otras se agruparon las carreras con menos de 5 estudiantes entrevistados.
\end{tabular}

CUADRO 3

Estudiantes matriculados en las Escuelas de la UNED por sexo, 2010

\begin{tabular}{lccc}
\hline \multicolumn{1}{c}{ Escuela } & $\begin{array}{c}\text { Mujeres } \\
(\%)\end{array}$ & $\begin{array}{c}\text { Hombres } \\
(\%)\end{array}$ & $\begin{array}{c}\text { Razón } \\
\text { M/H }\end{array}$ \\
\hline $\begin{array}{l}\text { Ciencias de la } \\
\text { Administración }\end{array}$ & 27,7 & 30,9 & 0,9 \\
$\begin{array}{l}\text { Ciencias Sociales y } \\
\text { Humanidades }\end{array}$ & 25,7 & 27,6 & 0,9 \\
$\begin{array}{l}\text { Ciencias Exactas y } \\
\text { Naturales }\end{array}$ & 16,4 & 33,0 & 0,5 \\
$\begin{array}{l}\text { Ciencias de la Educación } \\
\text { Total }\end{array}$ & 30,2 & 8,4 & 3,6 \\
& 100,0 & 100,0 & -
\end{tabular}

Fuente: Sánchez et al., 2010.

\section{Condiciones de estudio}

En educación a distancia, la tutoría presencial es un recurso provisto por la Universidad para apoyar el proceso educativo de la población estudiantil. En este caso, se presenta una brecha desfavorable para los hombres en la asistencia a tutorías, ya que la razón $\mathrm{M} / \mathrm{H}$ es de 1,4 en los casos de asistencia frecuente (siempre y regularmente).

En el acceso a internet, el cual es un recurso básico para un estudiante universitario en la modalidad a distancia, el $58,8 \%$ de las mujeres y el $43,5 \%$ de los hombres indican que cuentan con este servicio en sus casas; en este caso se presenta una brecha desfavorable para los hombres, ya que la razón es de 1,4 (Cuadro 4).

Respecto a la dedicación al estudio, de acuerdo con el número de horas que los/as estudiantes reportaron estudiar por semana, se obtiene que en su mayoría, tanto hombres como mujeres, lo hacen 10 horas o menos (Cuadro 5). Pero al analizar las relaciones entre sexos, se presenta una brecha que desfavorece a los hombres en la dedicación intensiva al estudio (21 o más horas), con una razón $\mathrm{M} / \mathrm{H}$ de 2,7 .

En su mayoría, el estudiantado tiene dependientes económicos o emocionales (73,3\% de las mujeres y $69,2 \%$ de los hombres) y esto repercute en dificultades para mantenerse en la UNED (58,3\% de las mujeres y $74,0 \%$ de los hombres).

Las principales dificultades para mantenerse estudiando en la UNED, para ambos sexos, fueron la carencia de recursos económicos y la falta de tiempo para estudiar e ir a tutorías debido a los compromisos laborales. Adicionalmente, en el caso de las mujeres se mencionaron el embarazo y el no contar con quien cuide los/as hijos/a.

En cuanto a los apoyos de la UNED para sus estudiantes, tales como becas, reposiciones de exámenes, información y facilidades para adecuar sus condiciones de

CUADRO 4

Asistencia del estudiantado a tutorías de la UNED por sexo, 2010

\begin{tabular}{lccc}
\hline \multicolumn{1}{c}{ Periodicidad } & $\begin{array}{c}\text { Mujeres } \\
(\%)\end{array}$ & $\begin{array}{c}\text { Hombres } \\
(\%)\end{array}$ & $\begin{array}{c}\text { Razón } \\
\text { M/H }\end{array}$ \\
\hline $\begin{array}{l}\text { Siempre o } \\
\text { regularmente }\end{array}$ & 63,6 & 44,2 & 1,4 \\
A veces o nunca & 36,4 & 55,8 & 0,7 \\
Total & 100,0 & 100,0 & -
\end{tabular}




\section{CUADRO 5}

Horas de estudio reportadas por estudiantes de la UNED, por sexo, 2010

$\begin{array}{lccc}\quad \text { Horas de estudio } & \text { Mujeres } & \text { Hombres } & \text { Razón M/H } \\ \text { 10 horas o menos } & 62,7 & 75,0 & 0,8 \\ \text { de 11 a 20 horas } & 21,7 & 19,2 & 1,1 \\ 21 \text { horas y más } & 15,7 & 5,8 & 2,7 \\ \text { Total } & 100,0 & 100,0 & -\end{array}$

estudio, se muestra una brecha favorable a las mujeres, ya que un $58,5 \%$ de las mujeres y un $42,9 \%$ de los hombres han obtenido este tipo de apoyos, lo cual arroja una razón $\mathrm{M} / \mathrm{H}$ de 1,36 .

\section{Percepciones y estereotipos de género}

La percepción sobre la igualdad de oportunidades de estudio para mujeres y hombres en la UNED es favorable en ambos sexos ( $88,4 \%$ mujeres y 84,6 hombres) y no hay brecha de género ya que la razón $\mathrm{M} / \mathrm{H}$ es de 1,0 .

Quienes respondieron que hay desigualdad manifiestan percepciones como las siguientes:

- Mayor facilidad del hombre para hacer frente al pago de la matrícula

- La ausencia de guarderías para las/os hijas/os de estudiantes

- Prioridad en las ayudas para las mujeres, tales como las becas, especialmente para quienes son madres.

- Carreras donde hay giras y laboratorios presentan mayores dificultades para las mujeres que para los hombres.

Un $50,0 \%$ de las mujeres y un $66,0 \%$ de los hombres consideran que no existe un trato diferenciado en la carrera que estudia, según sea el sexo de la persona estudiante, con lo cual al valorar la brecha de género se obtiene una razón $\mathrm{M} / \mathrm{H}$ de 0,8 . Entre las razones del estudiantado para justificar esta brecha se destaca que el contexto social y económico privilegia el que los hombres estudien ciertas carreras y las mujeres otras.

Hay un desconocimiento generalizado entre mujeres $(98,8 \%)$ y hombres $(90,4 \%)$ respecto a la existencia de medidas para reducir la discriminación de género entre estudiantes de la UNED; pero, apoyarían (79,1\% mujeres y $78,8 \%$ hombres) acciones contra las desigualdades de género. En este último aspecto, no se presenta brecha de género al obtenerse una razón $\mathrm{M} / \mathrm{H}$ de 1,0.
En cuanto a la afirmación "las mujeres tienen que cumplir con su rol tradicional de madre y cuidadora del hogar porque es su función natural", se manifestaron a favor un $10,5 \%$ de las mujeres y $17,3 \%$ de los hombres, lo cual arroja una razón $\mathrm{M} / \mathrm{H}$ de 0,6 y muestra una brecha de género. Además, el $30,2 \%$ de las mujeres y el $36,5 \%$ de los hombres consideran que estos últimos son "más fuertes, aventureros y valientes; mientras que las mujeres son más sensibles y románticas"; en este sentido, se presenta brecha de género ya que la razón $\mathrm{M} / \mathrm{H}$ es de 0,8 .

\section{Conocimiento sobre temas de género}

No hay mucha claridad en la diferenciación de los conceptos de sexo y género, ya que un $51,2 \%$ de las mujeres y un $42,3 \%$ de los hombres opinaron que son lo mismo o no saben, lo cual arroja una brecha al obtenerse una razón $\mathrm{M} / \mathrm{H}$ de 1,2 .

Respecto a la afirmación de si el género es cosa de mujeres $96,5 \%$ de ellas y el $94,2 \%$ de los hombres opinan que no es así, por lo tanto no hay brecha de género ya que la razón $\mathrm{M} / \mathrm{H}$ es de 1,0 .

\section{Resumen de brechas de género}

Una síntesis las brechas estudiadas se presentan en el Cuadro 6.

\section{DISCUSIÓN}

Como producto de la socialización del sistema educativo en general, se debe señalar que las mujeres construyen sus proyectos de vida eligiendo estudios orientados a ocupaciones, que a menudo son una prolongación de funciones asignadas en su rol de género, como por ejemplo las tareas de cuido, de educación, de protección de las demás personas, de servicio, de apoyo y de carácter asistencial (Sawnson \& Tokar, 1991; Luzzo, 1995; Bradley, 2000). Estos tipos de trabajo colocan a las mujeres en una posición de desventaja con respecto a los hombres, ya que estas labores tienen menor remuneración y prestigio social. Esta realidad la podemos observar claramente en la UNED cuando analizamos los porcentajes de hombres y mujeres que estudian en las diferentes escuelas y carreras, conformando unas más feminizadas y de menor estatus social y otras más masculinizadas de mayor prestigio.

Dado que la mayoría de las carreras que ofrece la UNED son tradicionalmente consideradas como femeninas (Ej: Preescolar, educación especial y educación en general; secretariado; salud y gerontología; enseñanza en diferentes 
CUADRO 6

Resumen de brechas de género en las oportunidades de estudio de la UNED

\begin{tabular}{lc}
\hline Brechas & Razón M/H \\
\hline Matrícula anual en la Escuela de Ciencias de la Administración & 0,9 \\
Matrícula anual en la Escuela de Ciencias Sociales y Humanidades & 0,9 \\
Matrícula anual en la Escuela de Ciencias Exactas y Naturales & 0,5 \\
Matrícula anual en la Escuela de Ciencias de la Educación & 3,6 \\
Asistencia con alta frecuencia a tutorías & 1,4 \\
Acceso a internet en la casa & 1,4 \\
Dedicación horaria intensiva al estudio & 2,7 \\
Percepción sobre igualdad de oportunidades de estudio para mujeres y hombres en la UNED & 1,0 \\
Percepción sobre no existencia de trato diferenciado en la carrera que estudia, según sea el & 0,8 \\
sexo de la persona estudiante & Percepción sobre el apoyo a medidas para reducir la discriminación de género entre \\
estudiantes de la UNED & 1,0 \\
Percepción del rol tradicional de los hombres y de las mujeres en la sociedad & 0,8 \\
Percepción de la mujer en su rol tradicional de cuido & 0,6 \\
Desconocimiento sobre los conceptos de sexo y género & 1,2 \\
Conocimiento de que el género no es sólo asunto de mujeres & 1,0
\end{tabular}

disciplinas y administración con énfasis en recursos humanos) y no se toman medidas para modificar los roles tradicionales de género que se encuentran detrás de cada una de ellas, el resultado es que tenemos una universidad con rostro femenino en la cual se reproducen las manifestaciones de las desigualdades de género que, además, se ven como normales en la vida cotidiana institucional y por lo tanto se invisibilizan constantemente.

Lo anterior se evidencia también al observar el desconocimiento sobre la diferencia entre los conceptos de sexo y género y las percepciones estereotipadas en las respuestas del estudiantado, donde las brechas de género muestran, además, que son los hombres quienes expresan en mayor medida esta perspectiva tradicional de los roles de género dado, posiblemente, a su posición de privilegio en la sociedad. No obstante, se observa que no existen brechas entre mujeres y hombres, por un lado, para apoyar las medidas específicas de reducción de la discriminación de género $y$, por otra parte, en considerar que género no se refiere solamente a las mujeres, lo cual representa un avance importante hacia el cambio cultural.

A pesar de que el modelo de la UNED no se percibe como excluyente de algún sexo, sino que se piensa que ambos gozan del mismo trato en la carrera que estudian y de la misma oportunidad de estudiar en esta institución, según la no existencia de brecha en esta última percepción, se pueden observar brechas desfavorables para los hombres -quienes son minoría en la UNED- en cuanto a la asistencia a las tutorías, al acceso de internet en la casa y en la dedicación horaria al estudio.

Para combatir estas brechas que afectan más a los hombres, se debe partir del análisis de que ellos registran jornadas de trabajo remunerado más intensivas que las mujeres, aunque en estas últimas se recargue más el trabajo doméstico no remunerado. En este punto es importante aclarar, que en general el estudiantado de la UNED es relativamente joven, pues casi dos terceras partes son menores de 30 años y se encuentran en estado de soltería; sin embargo, un alto porcentaje de ellos declara tener tanto 
dependientes emocionales como dependientes económicos. Por lo tanto, la población estudiantil que ingresa a la UNED tiene ciertas características que la hacen mirar esta institución como una opción de estudio dentro de sus posibilidades socioeconómicas.

Es preciso mencionar que se realizan importantes, pero aislados, esfuerzos por parte de diferentes personas e instancias de la Universidad, en solidaridad con la lucha por la equidad social y de género. Sin embargo, falta un mayor compromiso institucional hacia la incorporación de la perspectiva de género en las propuestas curriculares y en su institucionalización por medio de una estrategia de transversalización, con la rigurosidad teórica e instrumental que se requiere y con el personal especializado.

Lo anterior es fundamental para una real democratización de la enseñanza universitaria pues el sistema educativo en general, como ente socializador, tiene un rol protagónico en la transmisión de modelos sociales y en la reproducción de valores, normas, creencias, actitudes y estereotipos de género que se expresan de modo implícito y explícito en las nociones culturales que se transmiten por medio del currículum formal androcéntrico $y$, de manera invisible, por medio del currículum oculto de género. Este último, se refiere al ejercicio cotidiano, legitimado y considerado como normal en los procesos educativos, muchas veces inconsciente, de formas discriminatorias de relacionarse entre las personas, de transmisión de mensajes que refuerzan la superioridad y la autonomía de los hombres y la inferioridad y dependencia de las mujeres. Esto repercute en la autoconfianza de ellas, en su autoestima y en su proyecto de vida $y$, por otro lado, para los hombres que desean construir masculinidades no hegemónicas desarrolla diferentes correctivos -en ocasiones violentos, a veces no perceptibles- para que no modifiquen sus roles tradicionales (Munévar \& Villaseñor, 2005). Las universidades tienen la responsabilidad de contrarrestar lo anteriormente citado y evitar todas las formas de exclusión posibles (Palomar, 2005).

La transversalidad de género es tanto una estrategia metodológica como política, pues implica un posicionamiento diferente frente al quehacer universitario que requiere atención e investigación constante para identificar las diversas y ocultas (y no tan ocultas) formas de discriminación; es desarrollar altos grados de creatividad para combatirlas $y$, en fin, es una estrategia consciente de trabajo cotidiano hacia la igualdad y la equidad social.

Por ende, transversar género implica la movilización de todas las políticas generales y medidas particulares hacia el propósito específico de lograr la igualdad y la equidad, teniendo en cuenta activa y constantemente, la identificación y la planificación de sus posibles impactos diferenciados sobre los géneros. Estos impactos pueden afectar directa o indirectamente a mujeres y hombres y puede ser tanto de manera positiva como negativa; es decir, pueden conducir hacia la modificación de los roles tradicionales y la eliminación de las desigualdades o bien, reforzar y perpetuar las estructuras patriarcales que generan las desigualdades existentes entre los géneros.

Este cambio no se conseguirá ni fácil ni rápidamente, requerirá su tiempo e implicará desafíos para la cultura institucional en el desarrollo de la docencia, la investigación, la extensión y la gestión universitaria por medio de una Política Institucional de Igualdad y Equidad de Género y su respectivo plan de acción, como parte de la estrategia de institucionalización de la perspectiva de género en la Universidad.

En ese sentido, esta política significa el instrumento para articular las acciones y medidas del quehacer universitario en cuanto a incorporación de perspectiva de género se refiere, con el objetivo a mediano y largo plazo de eliminar las brechas de género y construir así el camino hacia la igualdad. La estrategia de institucionalización de este enfoque debe articular y definir las acciones adjudicadas a las distintas áreas, para avanzar en la construcción de capacidades internas contribuyendo al cambio organizacional sensible al género, el cual pasa no sólo por un cambio de valores sino también por un cambio de estructuras y procedimientos internos como resultado de un compromiso real con este propósito.

Este reto significa, además, promover un cambio en la cultura organizacional por medio de mecanismos participativos que involucren a todas las personas que integran la universidad como sujetos activos, ya que es a partir de la interacción articulada de cada uno y cada una que se logra la transformación cultural por una sociedad más justa e igualitaria.

\section{REFERENCIAS}

Bonder, G. (1994). Mujer y educación en América Latina: hacia la igualdad de oportunidades. Revista lberoamericana de Educación 6, 9-48.

Calvo, Y. (1990). A la mujerpor la palabra. Heredia, Costa Rica: EUNA.

Bradley, K. (2000). The incorporation of women into Higher Education: Paradoxical Outcomes? Sociology of Education 73, 1-18

Hausmann, R., Tyson, L. \& Zahidi, S. (2010). The global gender gap report 2010. Geneva, Suiza: World Economic Forum

Instituto Nacional de las Mujeres. (2002). Instrumento guía para otorgar el reconocimiento laboral a las organizaciones públicas y privadas de Costa Rica que desarrollen un Sistema de Gestión de Equidad de Género. San José, Costa Rica: GRAFOS. 
Lagarde, M. (1996). Género y feminismo: desarrollo humano y democracia. Madrid, España: Horas y Horas.

Mendenhall, W. (1987). Elementos de Muestreo. México: Grupo Editorial Iberoamérica.

Money, J. \& Tucker, P. (1978). Asignaturas sexuales. Barcelona, España: A.T.E.

Munévar, D. \& Villeseñor, M. (2005). Transversalidad de género. Una estrategia para el uso político-educativo de sus saberes. Revista de Estudios de Género La Ventana 21, 44-68.

Luzzo, D. (1995). Gender differences in college students career maturity and perceived barriers in career development. Journal of Counseling Development 73, 319-322.

Oakley A. (1972). Sex, gender and society. Londres, Inglaterra: Temple Smith.

Palomar, C. (2005). La política de género en la educación superior. Revista de Estudios de Género La Ventana 21, 7-43.
Rubin, G. (1975). The traffic in women: Notes on the political economy of sex. Reiter, R .(Ed). Toward an anthropology of women (pp.157-210), Nueva York, EEUU.: Monthly Review Press.

Sánchez, E., Zamora, T. \& Zúñiga, L. (2010). Anuario Estadístico 2010. Universidad Estatal a Distancia. Recuperado de http://www.uned.ac.cr/ciei1/estadisticas/documents/ Anuario2010.pdf

Stoller, R. (1968). Sex and gender. Nueva York, EEUU.: Science House.

Swanson, J.L. \& Tokar, D.M.(1991). College students' perceptions of barriers to career development. Journal of Vocational Behavior 38, 91-106.

The World Bank. (2011). The World development report 2012: Gender equality and development. Washington DC, EEUU.: The World Bank. 